\title{
Facile synthesis of novel benzotriazole derivatives and their antibacterial activities
}

\author{
JUN WAN $^{1,2}$, PENG-CHENG LV ${ }^{1}$, NA-NA TIAN ${ }^{2}$ and HAI-LIANG ZHU ${ }^{1, *}$ \\ ${ }^{1}$ State Key Laboratory of Pharmaceutical Biotechnology, Nanjing University, Nanjing, \\ Jiangsu 210093, P.R. China \\ ${ }^{2}$ Key Laboratory of Eco-chemical Engineering, Ministry of Education; College of Chemistry and \\ Molecular Engineering, Qingdao University of Science and Technology, Qingdao 266042, P.R. China \\ e-mail: zhuhl@nju.edu.cn
}

MS received 16 February 2009; revised 8 September 2009; accepted 14 October 2009

\begin{abstract}
A series of benzotriazole derivatives (compounds 1-27) were synthesized, and 24 (compounds 1-5, 9-27) of which were first reported. Their chemical structures were confirmed by means of ${ }^{1} \mathrm{H}$ NMR, IR and elemental analyses, coupled with one selected single crystal structure (compound 1). All the compounds were assayed for antibacterial activities against three Gram positive bacterial strains (Bacillus subtilis, Staphylococcus aureus and Streptococcus faecalis) and three Gram negative bacterial strains (Escherichia coli, Pseudomonas aeruginosa and Enterobacter cloacae) by MTT method. Among the compounds tested, most of them exhibited potent antibacterial activity against the six bacterial strains. Most importantly, compound 3-benzotriazol-1-yl-1-(4-bromo-phenyl)-2-[1,2,4]triazol-1-ylpropan-1-one (19) showed the most favourable antibacterial activity against $B$. subtilis, S. aureus, $S$. faecalis, P. aeruginosa, E. coli and E. cloacae with $\mathrm{MIC}$ of $1.56 \mu \mathrm{g} / \mathrm{mL}, 1.56 \mu \mathrm{g} / \mathrm{mL}, 1.56 \mu \mathrm{g} / \mathrm{mL}$, $3.12 \mu \mathrm{g} / \mathrm{mL}, 6.25 \mu \mathrm{g} / \mathrm{mL}$ and $6.25 \mu \mathrm{g} / \mathrm{mL}$, respectively.
\end{abstract}

Keywords. Benzotriazoles derivatives; antibacterial activities; crystal structure; structure-activity relationship.

\section{Introduction}

Heterocyclic compounds containing nitrogen atoms are considered to be one of the most effective antimicrobial drugs used either as single agents or in combination for cancer therapy.,2 Recent study showed that several benzotriazole and 1,2,4-triazole derivatives represented an interesting class of heterocycle $^{3}$ and became the most rapidly expanding group of antifungal compounds with the advantage of low toxicity, high oral bioavailability and broadspectrum activity. ${ }^{4-6}$ Moreover, a variety of benzotriazoles have been reported to inhibit the growth of some microorganisms and some benzotriazole derivatives show anti-inflammatory properties. ${ }^{7}$ In addition, Touami et al also reported that the conjugates of benzotriazole derivative photonucleases and DNA minor groove binders, exhibiting enhanced cleavage efficiency and unique selectivity. ${ }^{8}$ A class

\footnotetext{
*For correspondence
}

of stable benzotriazole esters was also reported as mechanism-based inactivators of SARS-3CL ${ }^{\text {pro }}$, which has been shown to be essential for replication of SARS virus. ${ }^{9}$ Besides, it has been proposed but not yet demonstrated that the benzotriazole derivatives have the effect on cancer development. ${ }^{10}$

$\mathrm{N}$-Substituted benzotriazoles exist as two isomers: $1 \mathrm{H}-$ and $2 \mathrm{H}$-substituted. It is generally agreed that $1 \mathrm{H}$-substituted dominated in solid and solution, whereas the proportion of the $2 \mathrm{H}$-tautomer increased in the gas phase. ${ }^{11}$ However, the energy difference between the two isomers is very little. ${ }^{12}$ Similarly, benzotriazoles containing Mannich bases have recently been synthesized also by amine exchange reactions, from the $N, N$-dimethylamino propiophenone hydrochlorides and benzotriazole, respectively. ${ }^{13}$ In this paper, 24 novel compounds containing $1 \mathrm{H}-$ benzotrizole were synthesized and screened for their antibacterial activities against three Gram positive bacterial strains (Bacillus subtilis, Staphylococcus aureus and Streptococcus faecalis) and three Gram 
negative bacterial strains (Escherichia coli, Pseudomonas aeruginosa and Enterobacter cloacae) by MTT method. Fortunately, we found that most compounds showed potent antibacterial activity against the six bacterial strains. Most importantly, compound 3-benzotriazol-1-yl-1-(4-bromo-phenyl)-2[1,2,4]triazol-1-yl-propan-1-one (19) showed the most favourable antibacterial activity against $B$. subtilis, S. aureus, S. faecalis, $P$. aeruginosa, E. coli and E. cloacae with MIC of $1.56 \mu \mathrm{g} / \mathrm{mL}$, $1.56 \mu \mathrm{g} / \mathrm{mL}, 1.56 \mu \mathrm{g} / \mathrm{mL}, 3.12 \mu \mathrm{g} / \mathrm{mL}, 6.25 \mu \mathrm{g} / \mathrm{mL}$ and $6.25 \mu \mathrm{g} / \mathrm{mL}$, respectively.

\section{Experimental}

\subsection{General}

${ }^{1} \mathrm{H}$ NMR was recorded on a $500 \mathrm{MHz}$ JEOL FX-90Q NMR spectrometer in $\mathrm{CDCl}_{3}$ as a solvent and with TMS as an internal standard. Melting points were measured on a Yanaco MP-500 melting point apparatus and were uncorrected. Elemental analyses were measured with a Vario EL III analyzer. IR spectra (4000-400 $\mathrm{cm}^{-1}$ ), as $\mathrm{KBr}$ pellets, were recorded on a Nicolet FT-IR 510P spectrophotometer. All reagents were obtained from commercial suppliers and were used without further purification.

\subsection{General procedure for the preparation of compounds}

Mannich bases were prepared by the Mannich reaction of substituted acetophenone and benzotriazole ${ }^{13}$ and two isomers, viz. $1 \mathrm{H}-$ and $2 \mathrm{H}$-substituted (scheme 1), were found in the products, with the former being the majority. The $1 \mathrm{H}-$ and $2 \mathrm{H}-$ substituted products were isolated by column chromatography. To a $250 \mathrm{~mL}$ of flask were added $0.02 \mathrm{~mol}$ of intermediate $1 \mathrm{H}$-substituted products in $50 \mathrm{~mL}$ of acetic acid and $0.04 \mathrm{~mol}$ of sodium acetate. Then $0.02 \mathrm{~mol}(3.2 \mathrm{~g})$ bromine was added dropwise with stirring at the room temperature. The reaction was maintained until the mixture was turned into colourless for about $4 \mathrm{~h}$. Then $50 \mathrm{~mL}$ of water and $20 \mathrm{~mL}$ of chloroform were added. Organic layer was successively washed with saturated sodium bicarbonate solution and brine, and then dried over with anhydrous magnesium sulfate to afford the intermediate. Triethylamine $(2.8 \mathrm{~mL}, 0.02 \mathrm{~mol})$ was added drop-wise to the stirring solution of intermediate $(0.02 \mathrm{~mol})$ and carboxylic acid $(0.02 \mathrm{~mol})$ in acetone at $0^{\circ} \mathrm{C}$. The mixture was stirred another $5 \mathrm{~h}$ in ice-water bath, then filtered and the filtrate concentrated gave a crude product which was poured into a saturated sodium chloride solution followed by extraction with chloroform. The combined organic extracts were washed with water, dried with $\mathrm{MgSO}_{4}$ and filtered. Removal of the solvent gave the residue, which was chromatographed on silica to afford the desired products of compounds 1-27.

2.2a Nicotinic acid 1-benzotriazol-1-ylmethyl-2(3-methoxy-phenyl)-2-oxo-ethyl ester (1): Eluent petroleum/ethyl acetate $(1: 1)$, Yield $77 \%$, pale yellow solid, mp: $122 \cdot 5-124 \cdot 0^{\circ} \mathrm{C}$; Anal. Calcd. (\%): C, 65.66; H, 4.51; N, 13.92; Found: C, 65.18; H, $4.36 ; \mathrm{N}, 13.99 ; \mathrm{IR}, v_{\max } / \mathrm{cm}^{-1}(\mathrm{KBr}): 1727,1688$ $(s, \mathrm{C}=\mathrm{O}), 1592(\mathrm{C}=\mathrm{N}), 1273,1090(s, \mathrm{C}-\mathrm{O}) ;{ }^{1} \mathrm{H}$ NMR $\left(\mathrm{CDCl}_{3}\right): 3.89\left(s, 3 \mathrm{H},-\mathrm{OCH}_{3}\right), 5.55,5.57$ $\left({ }^{2} J=15 ; \quad d, \quad 2 \mathrm{H}, \quad-\mathrm{CH}_{2}-\right), \quad 6 \cdot 88-6 \cdot 90 \quad\left({ }^{3} J=3 \cdot 8\right.$, $\left.{ }^{3} J=8.4 ; t, 1 \mathrm{H},-\mathrm{O}-\mathrm{CH}-\right), 7.43-9.06\left(m, 12 \mathrm{H}, \mathrm{H}_{\mathrm{Ar}}\right)$.

\section{2b Isonicotinic acid 1-benzotriazol-1-ylmethyl-2-} (3-methoxy-phenyl)-2-oxo-ethyl ester (2): Eluent using petroleum/ethyl acetate (2:1), Yield 47\%, pale yellow solid, mp: $143.5-145 \cdot 2^{\circ} \mathrm{C}$; Anal. Calcd. (\%): C, 65.66; H, 4.51; N, 13.92; Found: C, 65.71; H, 4.38; N, 13.53; IR, $v_{\max } / \mathrm{cm}^{-1}(\mathrm{KBr}): 1733$, $1698(s, \mathrm{C}=\mathrm{O}), 1581(\mathrm{C}=\mathrm{N}), 1287,1127(s, \mathrm{C}-\mathrm{O})$; ${ }^{1} \mathrm{H}$ NMR $\left(\mathrm{CDCl}_{3}\right): 3.95\left(s, 3 \mathrm{H},-\mathrm{OCH}_{3}\right), 5.54,5.57$ $\left({ }^{2} \mathrm{~J}=14.9 ; \quad d, \quad 2 \mathrm{H}, \quad-\mathrm{CH}_{2}-\right), \quad 6 \cdot 88-6.90 \quad\left({ }^{3} \mathrm{~J}=3 \cdot 8\right.$, $\left.{ }^{3} J=8 \cdot 5 ; t, 1 \mathrm{H},-\mathrm{O}-\mathrm{CH}-\right), 7 \cdot 30-8.01\left(m, 12 \mathrm{H}, \mathrm{H}_{\mathrm{Ar}}\right)$.

2.2c Benzoic acid 1-benzotriazol-1-ylmethyl-2-(3methoxy-phenyl)-2-oxo-ethyl ester (3): Eluent ethanol, Yield 35\%, white solid, m.p.: 150.0$152.0^{\circ} \mathrm{C}$; Anal. Calcd. (\%): C, 68.82; H, 4.77; N, 10.47; Found: C, 68.86; H, 4.89; N, 10.08; IR, $\nu_{\max } / \mathrm{cm}^{-1}(\mathrm{KBr}): 1717,1688(\mathrm{~s}, \mathrm{C}=\mathrm{O}), 1595(\mathrm{C}=\mathrm{N})$, $1271,1123(s, \mathrm{C}-\mathrm{O}) ;{ }^{1} \mathrm{H}$ NMR $\left(\mathrm{CDCl}_{3}\right): 4 \cdot 15(s, 3 \mathrm{H}$, $\left.-\mathrm{OCH}_{3}\right), 5.58,5.89 \quad\left({ }^{2} \mathrm{~J}=14.95 ; d, 2 \mathrm{H},-\mathrm{CH}_{2}-\right)$, $6.69-6.71 \quad\left({ }^{3} J=3.75,{ }^{3} J=8.3 ; \quad t, \quad 1 \mathrm{H}, \quad-\mathrm{O}-\mathrm{CH}-\right)$, 7.02-7.82 (m, 13H, $\left.\mathrm{H}_{\mathrm{Ar}}\right)$.

\section{2d 2,4-Dichloro-benzoic acid 1-benzotriazol-1-} ylmethyl-2-(3-methoxy-phenyl)-2-oxo-ethyl ester (4): Eluent ethanol, Yield 55\%, white solid, m.p.: $148.5-150.0^{\circ} \mathrm{C}$; Anal. Calcd. (\%): C, 58.74; H, 3.64; N, 8.93; Found: C, 58.47; H, 3.47; N, 8.89; IR, $\nu_{\max } / \mathrm{cm}^{-1}(\mathrm{KBr}): 1751,1695(\mathrm{~s}, \mathrm{C}=\mathrm{O}), 1584(\mathrm{C}=\mathrm{N})$, 1260, $1082(s, \mathrm{C}-\mathrm{O}) ;{ }^{1} \mathrm{H}$ NMR $\left(\mathrm{CDCl}_{3}\right): 3.83(s, 3 \mathrm{H}$, $\left.-\mathrm{OCH}_{3}\right), 5.43,5.45 \quad\left({ }^{2} \mathrm{~J}=14.85 ; d, 2 \mathrm{H},-\mathrm{CH}_{2}-\right)$, 
$6.62-6.82 \quad\left({ }^{3} J=3.75, \quad{ }^{3} J=8 \cdot 4 ; \quad t, \quad 1 \mathrm{H}, \quad-\mathrm{O}-\mathrm{CH}-\right)$, $7 \cdot 25-7 \cdot 67\left(m, 11 \mathrm{H}, \mathrm{H}_{\mathrm{Ar}}\right)$.

2.2e 2-Chloro-benzoic acid 1-benzotriazol-1ylmethyl-2-(3-methoxy-phenyl)-2-oxo-ethyl ester (5): Eluent ethanol, Yield 46\%, white solid, m.p.: $155.4-157 \cdot 0^{\circ} \mathrm{C}$; Anal. Calcd. (\%): C, 63.38; H, $4 \cdot 16$; N, 9.64; Found: C, 63.08; H, 4.11; N, 9.43; IR, $v_{\max } / \mathrm{cm}^{-1}(\mathrm{KBr}): 1706,1687(s, \mathrm{C}=\mathrm{O}), 1591(\mathrm{C}=\mathrm{N})$, 1271, $1110(s, \mathrm{C}-\mathrm{O}) ;{ }^{1} \mathrm{H}$ NMR $\left(\mathrm{CDCl}_{3}\right): 3.90(s, 3 \mathrm{H}$, $\left.-\mathrm{OCH}_{3}\right), 5 \cdot 50,5.52\left({ }^{2} \mathrm{~J}=15 ; d, 2 \mathrm{H},-\mathrm{CH}_{2}-\right), 6 \cdot 88-$ $6.90\left({ }^{3} \mathrm{~J}=3 \cdot 7,{ }^{3} \mathrm{~J}=8 \cdot 5 ; t, 1 \mathrm{H},-\mathrm{O}-\mathrm{CH}-\right), 7.41-7.90$ $\left(m, 12 \mathrm{H}, \mathrm{H}_{\mathrm{Ar}}\right)$.

2.2f Benzoic acid 1-benzotriazol-1-ylmethyl-2oxo-2-p-tolyl-ethyl ester (6). 2,4-Dichloro-benzoic acid 1-benzotriazol-1-ylmethyl-2-oxo-2-p-tolyl-ethyl ester (7). 4-Ethyl-benzoic acid 1-benzotriazol-1ylmethyl-2-oxo-2-p-tolyl-ethyl ester (8). 4-Chlorobenzoic acid 1-benzotriazol-1-ylmethyl-2-oxo-2-ptolyl-ethyl ester (9): Eluent petroleum/ethyl acetate, Yield $42 \%$, white solid, m.p.: 150.6$151.2^{\circ} \mathrm{C}$; Anal. Calcd. (\%): C, 65.79; H, 4.32; N, 10.01; Found: C, 65.41; H, 4.23; N, 10.31; IR, $\nu_{\max } / \mathrm{cm}^{-1}(\mathrm{KBr}): 1723,1690(s, \mathrm{C}=\mathrm{O}), 1606(\mathrm{C}=\mathrm{N})$, 1267, $1096(s, \mathrm{C}-\mathrm{O}) ;{ }^{1} \mathrm{H}$ NMR $\left(\mathrm{CDCl}_{3}\right): 2.46(s, 3 \mathrm{H}$, $\left.\mathrm{CH}_{3}\right), \quad 5 \cdot 19, \quad 5.32 \quad\left({ }^{2} \mathrm{~J}=15 ; \quad d, \quad 2 \mathrm{H}, \quad \mathrm{CH}_{2}\right), \quad 6.64$ $\left({ }^{3} J=8 \cdot 5 ; 1 \mathrm{H}, \mathrm{CH}\right), 7 \cdot 28-7.95\left(12 \mathrm{H}, \mathrm{H}_{\mathrm{Ar}}\right)$.

$2.2 \mathrm{~g}$ 2-Chloro-benzoic acid 1-benzotriazol-1ylmethyl-2-oxo-2-p-tolyl-ethyl ester (10): Eluent petroleum/ethyl acetate, Yield $40 \%$, white solid, m.p.: $148.0-149 \cdot 2^{\circ} \mathrm{C}$; Anal. Calcd. (\%): C, 65.79; $\mathrm{H}, 4.32 ; \mathrm{N}, 10.01$; Found: C, 65.33; H, 4.36; N, 10.23 ; IR, $v_{\max } / \mathrm{cm}^{-1}(\mathrm{KBr}): 1730,1693(s, \mathrm{C}=\mathrm{O})$, $1605 \quad(\mathrm{C}=\mathrm{N}), \quad 1300,1130 \quad(s, \mathrm{C}-\mathrm{O}) ;{ }^{1} \mathrm{H} \quad \mathrm{NMR}$ $\left(\mathrm{CDCl}_{3}\right): 2.43\left(s, 3 \mathrm{H}, \mathrm{CH}_{3}\right), 5 \cdot 16,5 \cdot 26\left({ }^{2} J=14.95\right.$; $\left.d, 2 \mathrm{H}, \mathrm{CH}_{2}\right), 6.68\left({ }^{3} J=8.3 ; 1 \mathrm{H}, \mathrm{CH}\right), 7.26-8.05$ $\left(12 \mathrm{H}, \mathrm{H}_{\mathrm{Ar}}\right)$.

$2.2 \mathrm{~h}$ 2-Chloro-benzoic acid 1-benzotriazol-1ylmethyl-2-(3-chloro-phenyl)-2-oxo-ethyl ester (11): Eluent acetone/ethanol, Yield 44\%, white solid, m.p.: $130.0-131.0^{\circ} \mathrm{C}$; Anal. Calcd. (\%): C, 60.02; $\mathrm{H}, 3.43 ; \mathrm{N}, 9.54$; Found: C, 60.34; H, 3.18; N, 10.08; IR, $v_{\max } / \mathrm{cm}^{-1}(\mathrm{KBr}): 1737,1701 \quad(s, \mathrm{C}=\mathrm{O})$, $1593(\mathrm{C}=\mathrm{N}), 1230,1126 \quad(s, \mathrm{C}-\mathrm{O}) ;{ }^{1} \mathrm{H} \quad \mathrm{NMR}$ $\left(\mathrm{CDCl}_{3}\right): 5 \cdot 47,5 \cdot 51\left({ }^{2} \mathrm{~J}=14.5 ; d, 2 \mathrm{H},-\mathrm{CH}_{2}-\right), 6 \cdot 82-$ $6.84\left({ }^{3} \mathrm{~J}=3.75,{ }^{3} \mathrm{~J}=8.4 ; t, 1 \mathrm{H},-\mathrm{O}-\mathrm{CH}-\right), 7.33-7.68$ $\left(m, 12 \mathrm{H}, \mathrm{H}_{\mathrm{Ar}}\right)$.

2.2i Isonicotinic acid 1-benzotriazol-1-ylmethyl-2(3-chloro-phenyl)-2-oxo-ethyl ester (12): Eluent petroleum/ethyl acetate $(3: 1)$ as the eluent, Yield $48 \%$, pale yellow solid, m.p.: $128.0-130 \cdot 0^{\circ} \mathrm{C}$; Anal. Calcd. (\%): C, 62.00; H, 3.72; N, 13.77; Found: $\mathrm{C}, 62 \cdot 12 ; \mathrm{H}, 3.68 ; \mathrm{N}, 13.56, \mathrm{IR}, v_{\max } / \mathrm{cm}^{-1}$ $(\mathrm{KBr}): 1741,1694(\mathrm{C}=\mathrm{O}), 1591(\mathrm{C}=\mathrm{N}), 1230,1120$ $(\mathrm{C}-\mathrm{O}) ;{ }^{1} \mathrm{H}$ NMR $\left(\mathrm{CDCl}_{3}\right): 5 \cdot 58-5 \cdot 60\left({ }^{2} J=15 ; 2 \mathrm{H}\right.$, $\left.\mathrm{CH}_{2}\right), \quad 6.90-6.92 \quad\left({ }^{3} J=8.3 ; \quad 1 \mathrm{H}, \quad \mathrm{CH}\right), \quad 7.43-8.11$ $\left(10 \mathrm{H}, \mathrm{H}_{\mathrm{Ar}}\right), 8 \cdot 79-8 \cdot 81\left(2 \mathrm{H}, \mathrm{H}_{\mathrm{py}}\right)$.

$2.2 \mathrm{j}$ Nicotinic acid 1-benzotriazol-1-ylmethyl-2-(4bromo-phenyl)-2-oxo-ethyl ester (13): Eluent petroleum/ethyl acetate $(1: 1)$ as the eluent, Yield $36 \%$, pale yellow solid, m.p.: $157.5-159 \cdot 0^{\circ} \mathrm{C}$; Anal. Calcd. (\%): C, 55.89; H, 3.35; N, 12.42; Found: C, 55.82; H, 3.27; N, 11.92; IR, $v_{\max } / \mathrm{cm}^{-1}$ $(\mathrm{KBr}): 1728,1692(s, \mathrm{C}=\mathrm{O}), 1586(\mathrm{C}=\mathrm{N}), 1285$, $1109(s, \mathrm{C}-\mathrm{O}) ;{ }^{1} \mathrm{H}$ NMR $\left(\mathrm{CDCl}_{3}\right): 5.68,5.71$ $\left({ }^{2} J=14.75 ; \quad d, 2 \mathrm{H},-\mathrm{CH}_{2}-\right), 6.99-7.00 \quad\left({ }^{3} J=3.7\right.$, $\left.{ }^{3} J=8.5 ; t, 1 \mathrm{H},-\mathrm{O}-\mathrm{CH}-\right), 7 \cdot 55-9 \cdot 18\left(m, 12 \mathrm{H}, \mathrm{H}_{\mathrm{Ar}}\right)$.

$2.2 \mathrm{k}$ Isonicotinic acid 1-benzotriazol-1-ylmethyl-2(4-bromo-phenyl)-2-oxo-ethyl ester (14): Eluent using petroleum/ethyl acetate $(2: 1)$ as the eluent, Yield 42\%, pale yellow solid, m.p.: 158.5$159.5^{\circ} \mathrm{C}$; Anal. Calcd. (\%): C, 55.89; H, 3.35; N, 12.42; Found: C, 55.68; H, 3.25; N, 11.99; IR, $v_{\max } / \mathrm{cm}^{-1}(\mathrm{KBr}): 1736,1702(s, \mathrm{C}=\mathrm{O}), 1586(\mathrm{C}=\mathrm{N})$, 1269, $1100(s, \mathrm{C}-\mathrm{O}) ;{ }^{1} \mathrm{H}$ NMR $\left(\mathrm{CDCl}_{3}\right): 5.40,5.43$ $\left({ }^{2} J=15 ; \quad d, \quad 2 \mathrm{H}, \quad-\mathrm{CH}_{2}-\right), 6.72-6.74 \quad\left({ }^{3} J=3.75\right.$, $\left.{ }^{3} J=8.43 ; t, 1 \mathrm{H},-\mathrm{O}-\mathrm{CH}-\right), 7.29-8.62\left(m, 12 \mathrm{H}, \mathrm{H}_{\mathrm{Ar}}\right)$.

2.21 4-Chloro-benzoic acid 1-benzotriazol-1ylmethyl-2-(4-bromo-phenyl)-2-oxo-ethyl ester (15): Eluent ethanol, Yield 50\%, white solid, m.p.: $159.5-161.5^{\circ} \mathrm{C}$; Anal. Calcd. (\%): C, 54.51; H, $3 \cdot 12$; N, 8.67; Found: C, 54.51; H, 3.11; N, 8.62; IR, $v_{\max } / \mathrm{cm}^{-1}(\mathrm{KBr})$ : $1720,1687(\mathrm{~s}, \mathrm{C}=\mathrm{O}), 1589(\mathrm{C}=\mathrm{N})$, 1255, $1077(s, \mathrm{C}-\mathrm{O}) ;{ }^{1} \mathrm{H}$ NMR $\left(\mathrm{CDCl}_{3}\right): 5.52,5.55$ $\left({ }^{2} J=15 ; d, 2 \mathrm{H}, \mathrm{CH}_{2}\right), 6 \cdot 82\left({ }^{3} J=8 \cdot 3 ; 1 \mathrm{H}, \mathrm{CH}\right), 7.53-$ $7.97(12 \mathrm{H}, \mathrm{HAr})$.

$2.2 \mathrm{~m}$ 2-Chloro-benzoic acid 1-benzotriazol-1ylmethyl-2-(4-bromo-phenyl)-2-oxo-ethyl ester (16): Eluent ethanol, Yield $48 \%$, white solid, m.p.: $164.5-166.0^{\circ} \mathrm{C}$; Anal. Calcd. (\%):C, 54.51; H, $3 \cdot 12$; N, 8.67; Found: C, 54.43; H, 3.09; N, 8.69; IR, $v_{\max } / \mathrm{cm}^{-1}(\mathrm{KBr}): 1736,1692(s, \mathrm{C}=\mathrm{O}), 1587(\mathrm{C}=\mathrm{N})$, 1248, $1087(s, \mathrm{C}-\mathrm{O}) ;{ }^{1} \mathrm{H}$ NMR $\left(\mathrm{CDCl}_{3}\right): 5 \cdot 50,5 \cdot 52$ $\left({ }^{2} J=14.75 ; d, 2 \mathrm{H}, \mathrm{CH}_{2}\right), 6.86\left({ }^{3} J=8.4 ; 1 \mathrm{H}, \mathrm{CH}\right)$, 7.40-7.92 (12H, HAr).

2.2n 4-Methyl-benzoic acid 1-benzotriazol-1ylmethyl-2-(4-bromo-phenyl)-2-oxo-ethyl ester (17): 
Eluent acetone/ethanol, Yield 45\%, white solid, m.p.: $176 \cdot 0-178 \cdot 0^{\circ} \mathrm{C}$; Anal. Calcd. (\%): C, 59.50; H, 3.91; N, 9.05; Found(\%): C, 59.73; H, 3.82; N, 8.97; IR, $v_{\max } / \mathrm{cm}^{-1}(\mathrm{KBr}): 1723,1695(s, \mathrm{C}=\mathrm{O})$, $1575(\mathrm{C}=\mathrm{N}), 1247,1106(s, \mathrm{C}-\mathrm{O}) ;{ }^{1} \mathrm{H}$ NMR $\left(\mathrm{CDCl}_{3}\right)$ : 2.39 (3H, methyl, $\left.\mathrm{CH}_{3}\right), 5.46,5.55\left({ }^{2} J=14.95 ; 2 \mathrm{H}\right.$, $\left.\mathrm{CH}_{2}\right), 6.76 \quad\left({ }^{3} \mathrm{~J}=8.5 ; 1 \mathrm{H}, \mathrm{CH}\right), 7.28-7.99(12 \mathrm{H}$, $\mathrm{H}_{\mathrm{Ar}}$ ).

2.2o 2-Methyl-benzoic acid 1-benzotriazol-1ylmethyl-2-(4-bromo-phenyl)-2-oxo-ethyl ester (18): acetone/ethanol, Yield 44\%, white solid, m.p.: $179.5-181.0^{\circ} \mathrm{C}$; Anal. Calcd. (\%): C, 59.50; H, $3.91 ; \mathrm{N}, 9.05$; Found (\%): C, 59.37; H, 3.84; N, 8.87; IR, $v_{\max } / \mathrm{cm}^{-1}(\mathrm{KBr}): 1719,1688(s, \mathrm{C}=\mathrm{O})$, $1587 \quad(\mathrm{C}=\mathrm{N}), 1253,1077 \quad(s, \mathrm{C}-\mathrm{O}) ;{ }^{1} \mathrm{H}$ NMR $\left(\mathrm{CDCl}_{3}\right): 2.39 \quad\left(3 \mathrm{H}\right.$, methyl, $\left.\mathrm{CH}_{3}\right), 5.47,5.55$ $\left({ }^{2} J=14.85 ; 2 \mathrm{H}, \mathrm{CH}_{2}\right), 6.77 \quad\left({ }^{3} J=8.3 ; 1 \mathrm{H}, \mathrm{CH}\right)$, $7 \cdot 28-7.81\left(12 \mathrm{H}, \mathrm{H}_{\mathrm{Ar}}\right)$.

\section{$2.2 \mathrm{p}$ 3-Benzotriazol-1-yl-1-(4-bromo-phenyl)-2-}

[1,2,4]triazol-1-yl-propan-1-one (19): Eluent petroleum/ethyl acetate $(1: 1)$ as the eluent, Yield $32 \%$, white solid, mp: $180 \cdot 5-182 \cdot 0^{\circ} \mathrm{C}$; Anal. Calcd. (\%): C, 51.40; H, 3.30; N, 21.16; Found (\%): C, 51.26; H, $3.41 ; \mathrm{N}, 20.97$; IR, $v_{\max } / \mathrm{cm}^{-1}(\mathrm{KBr}): 1708(\mathrm{~s}, \mathrm{C}=\mathrm{O})$, $1585(\mathrm{C}=\mathrm{N}) ; \quad{ }^{1} \mathrm{H} N M R \quad\left(\mathrm{CDCl}_{3}\right): \quad 4.45, \quad 4.54$ $\left({ }^{2} J=14.85 ; 2 \mathrm{H}, \quad \mathrm{CH}_{2}\right), 5.69 \quad\left({ }^{3} J=8.4 ; 1 \mathrm{H}, \mathrm{CH}\right)$, $7 \cdot 35-8 \cdot 24\left(8 \mathrm{H}, \mathrm{H}_{\mathrm{Ar}}\right), 8 \cdot 24,8 \cdot 46\left(2 \mathrm{H}, \mathrm{H}_{\mathrm{Tr}}\right)$.

2.2q Nicotinic acid 1-benzotriazol-1-ylmethyl-2(2-methyl-phenyl)-2-oxo-ethyl ester (20): Eluent petroleum/ethyl acetate $(1: 1)$ as the eluent, Yield $39 \%$, pale yellow solid, m.p.: $145 \cdot 5-147 \cdot 0^{\circ} \mathrm{C}$; Anal. Calcd. (\%): C, 68.38; H, 4.70; N, 14.50; Found: C, 68.18; H, 4.65; N, 14.44; IR, $v_{\max } / \mathrm{cm}^{-1}$ (KBr): 1731, $1694(s, \mathrm{C}=\mathrm{O}), 1593(\mathrm{C}=\mathrm{N}), 1286$, $1105(s, \mathrm{C}-\mathrm{O}) ;{ }^{1} \mathrm{H}$ NMR $\left(\mathrm{CDCl}_{3}\right): 2.40\left(3 \mathrm{H}, \mathrm{CH}_{3}\right)$, $5.44,5.43\left({ }^{2} J=15 ; d, 2 \mathrm{H},-\mathrm{CH}_{2}-\right), 6.75 \quad\left({ }^{3} J=3.7\right.$, $\left.{ }^{3} J=8.43 ; t, 1 \mathrm{H},-\mathrm{O}-\mathrm{CH}-\right), 7.33-8.93\left(m, 12 \mathrm{H}, \mathrm{H}_{\mathrm{Ar}}\right)$.

2.2r Isonicotinic acid 1-benzotriazol-1-ylmethyl-2(2-methyl phenyl)-2-oxo-ethyl ester (21): Eluent petroleum/ethyl acetate $(2: 1)$ as the eluent, Yield $35 \%$, pale yellow solid, m.p.: $143.5-145.0^{\circ} \mathrm{C}$; Anal. Calcd.(\%): C, 68.38; H, 4.70; N, 14.50; Found: C, 68.10; H, 4.98; N, 14.69; IR, $v_{\max } / \mathrm{cm}^{-1}$ (KBr): 1739, $1688(s, \mathrm{C}=\mathrm{O}), 1599(\mathrm{C}=\mathrm{N}), 1278$, $1086(s, \mathrm{C}-\mathrm{O}) ;{ }^{1} \mathrm{H}$ NMR $\left(\mathrm{CDCl}_{3}\right): 2.50\left(3 \mathrm{H}, \mathrm{CH}_{3}\right)$, $5.25, \quad 5.24 \quad\left({ }^{2} J=14.95 ; \quad d, \quad 2 \mathrm{H}, \quad-\mathrm{CH}_{2}-\right), \quad 6.57$ $\left({ }^{3} J=3.75,{ }^{3} J=8.43 ; t, 1 \mathrm{H},-\mathrm{O}-\mathrm{CH}-\right), 7 \cdot 14-8.51(\mathrm{~m}$, $\left.12 \mathrm{H}, \mathrm{H}_{\mathrm{Ar}}\right)$. 2.2s 4-Chloro-benzoic acid 1-benzotriazol-1ylmethyl-2-(2-methyl-phenyl)-2-oxo-ethyl ester (22): ethanol, Yield 48\%, white solid, m.p.: $129.5-$ $131.5^{\circ} \mathrm{C}$; Anal. Calcd. (\%): C, 65.79; H, 4.32; N, 10.01; Found: $\mathrm{C}, 65.51 ; \mathrm{H}, 4.11$; N, 9.82; IR, $v_{\max } / \mathrm{cm}^{-1}(\mathrm{KBr}): 1724,1693(s, \mathrm{C}=\mathrm{O}), 1586(\mathrm{C}=\mathrm{N})$, 1228, $1106(s, \mathrm{C}-\mathrm{O}) ;{ }^{1} \mathrm{H}$ NMR $\left(\mathrm{CDCl}_{3}\right): 2.60(3 \mathrm{H}$, $\left.\mathrm{CH}_{3}\right), 5.42,5.71 \quad\left({ }^{2} \mathrm{~J}=14.75 ; d, 2 \mathrm{H}, \mathrm{CH}_{2}\right), 6.32$ $\left({ }^{3} J=8 \cdot 5 ; 1 \mathrm{H}, \mathrm{CH}\right), 7 \cdot 49-8 \cdot 17\left(12 \mathrm{H}, \mathrm{H}_{\mathrm{Ar}}\right)$.

2.2t 2-Chloro-benzoic acid 1-benzotriazol-1ylmethyl-2-(2-methyl-phenyl)-2-oxo-ethyl ester (23): ethanol, Yield 47\%, white solid, m.p.: 118.5$120.0^{\circ} \mathrm{C}$; Anal. Calcd. (\%): C, 65.79; H, 4.32; N, 10.01; Found: C, 66.04; H, 4.16; N, 9.84; IR, $v_{\max } / \mathrm{cm}^{-1}(\mathrm{KBr}): 1726,1696(s, \mathrm{C}=\mathrm{O}), 1588(\mathrm{C}=\mathrm{N})$, 1227, $1107(s, \mathrm{C}-\mathrm{O}) ;{ }^{1} \mathrm{H}$ NMR $\left(\mathrm{CDCl}_{3}\right): 2.40(3 \mathrm{H}$, $\left.\mathrm{CH}_{3}\right), 5.39,5.41 \quad\left({ }^{2} J=14.95 ; d, 2 \mathrm{H}, \mathrm{CH}_{2}\right), 6.73$ $\left({ }^{3} J=8 \cdot 3 ; 1 \mathrm{H}, \mathrm{CH}\right), 7 \cdot 30-7.58\left(12 \mathrm{H}, \mathrm{H}_{\mathrm{Ar}}\right)$.

2.2u 4-Methyl-benzoic acid 1-benzotriazol-1ylmethyl-2-(2-methyl-phenyl)-2-oxo-ethyl ester (24): Eluent acetone, Yield 48\%, white solid, m.p.: $117.0-119.5^{\circ} \mathrm{C}$; Anal. Calcd. (\%): C, 72.16; H, $5.30 ; \mathrm{N}, 10.52$; Found (\%): C, 72.18; H, 5.13; N, 10.46; IR, $v_{\max } / \mathrm{cm}^{-1}(\mathrm{KBr}): 1720,1696(s, \mathrm{C}=\mathrm{O})$, $1610 \quad(\mathrm{C}=\mathrm{N}), \quad 1291,1102 \quad(s, \mathrm{C}-\mathrm{O}) ;{ }^{1} \mathrm{H}$ NMR $\left(\mathrm{CDCl}_{3}\right): 2.34-2.46\left(6 \mathrm{H}\right.$, methyl, $\left.\mathrm{CH}_{3}\right), 5.44,5.45$ $\left({ }^{2} J=15 ; 2 \mathrm{H}, \mathrm{CH}_{2}\right), 6.69\left({ }^{3} J=8.4 ; 1 \mathrm{H}, \mathrm{CH}\right), 7 \cdot 22-$ $7.96\left(12 \mathrm{H}, \mathrm{H}_{\mathrm{Ar}}\right)$.

2.2v 4-Ethyl-benzoic acid 1-benzotriazol-1ylmethyl-2-(2-methyl-phenyl)-2-oxo-ethyl ester (25): Eluent acetone, Yield 42\%, white solid, m.p.: $111.2-114.2{ }^{\circ} \mathrm{C}$; Anal. Calcd. (\%): C, 72.62; H, $5.61 ; \mathrm{N}, 10.16$; Found (\%): C, 73.09; H, 5.83; N, 9.96; IR, $v_{\max } / \mathrm{cm}^{-1}(\mathrm{KBr}): 1719,1697(s, \mathrm{C}=\mathrm{O})$, $1609(\mathrm{C}=\mathrm{N}), 1292,1096(s, \mathrm{C}-\mathrm{O}) ;{ }^{1} \mathrm{H}$ NMR $\left(\mathrm{CDCl}_{3}\right)$ : 1.19 (3H, ethyl, $\left.\mathrm{CH}_{3}\right), 2.45$ (3H, methyl, $\left.\mathrm{CH}_{3}\right), 2 \cdot 80$ $\left(2 \mathrm{H}\right.$, ethyl, $\left.\mathrm{CH}_{2}\right), 5.45,5.54\left({ }^{2} J=14.75 ; 2 \mathrm{H}, \mathrm{CH}_{2}\right)$, $6.69\left({ }^{3} J=8.5 ; 1 \mathrm{H}, \mathrm{CH}\right), 7 \cdot 27-7.99\left(12 \mathrm{H}, \mathrm{H}_{\mathrm{Ar}}\right)$.

2.2w 2-Methyl-benzoic acid 1-benzotriazol-1ylmethyl-2-(2-methyl-phenyl)-2-oxo-ethyl ester (26): ethanol, Yield 43\%, white solid, m.p.: 121.5123. $0^{\circ} \mathrm{C}$; Anal. Calcd. (\%): C, 72.16; H, 5.30; N, 10.52; Found (\%): C, 72.18; H, 5.13; N, 10.41; IR, $v_{\max } / \mathrm{cm}^{-1}(\mathrm{KBr}): 1720,1693(s, \mathrm{C}=\mathrm{O}), 1610(\mathrm{C}=\mathrm{N})$, 1292, $1101(\mathrm{~s}, \mathrm{C}-\mathrm{O}) ;{ }^{1} \mathrm{H}$ NMR $\left(\mathrm{CDCl}_{3}\right): 2 \cdot 36-2 \cdot 45$ $\left(6 \mathrm{H}\right.$, methyl, $\left.\mathrm{CH}_{3}\right), 5.43,5.45\left({ }^{2} J=15 ; 2 \mathrm{H}, \mathrm{CH}_{2}\right)$, $6.69\left({ }^{3} J=8 \cdot 5 ; 1 \mathrm{H}, \mathrm{CH}\right), 7 \cdot 23-7.45\left(12 \mathrm{H}, \mathrm{H}_{\mathrm{Ar}}\right)$. 
2.2x 3-Benzotriazol-1-yl-1-(2-methyl-phenyl)-2[1,2,4]triazol-1-yl-propan-1-one (27): petroleum/ ethyl acetate $(1: 1)$ as the eluent, Yield $28 \%$, white solid, m.p.: $109 \cdot 5-111 \cdot 0^{\circ} \mathrm{C}$; Anal. Calcd. (\%): C, 65.05; H, 4.85; N, 25.26; Found (\%): C, 65.29; H, $4.88 ; \mathrm{N}, 25 \cdot 35 ; \mathrm{IR}, v_{\max } / \mathrm{cm}^{-1}(\mathrm{KBr}): 1691(s, \mathrm{C}=\mathrm{O})$, $1595(\mathrm{C}=\mathrm{N}), 1251,1147 \quad(s, \mathrm{C}-\mathrm{O}) ;{ }^{1} \mathrm{H} \quad \mathrm{NMR}$ $\left(\mathrm{CDCl}_{3}\right): 2.38 \quad\left(3 \mathrm{H}\right.$, methyl, $\left.\mathrm{CH}_{3}\right), 5.43,5.51$ $\left({ }^{2} J=14.95 ; 2 \mathrm{H}, \quad \mathrm{CH}_{2}\right), 6 \cdot 17 \quad\left({ }^{3} J=8.4 ; \quad 1 \mathrm{H}, \mathrm{CH}\right)$, 7.29-7.56 $\left(8 \mathrm{H}, \mathrm{H}_{\mathrm{Ar}}\right), 7.90-7.94\left(2 \mathrm{H}, \mathrm{H}_{\mathrm{Tr}}\right)$.

\subsection{Crystallographic study}

Crystal data were collected at low temperatures using a BRUKER SMART 1000 CCD area diffractormeter. Empirical absorption correction was carried out by using the SADABS ${ }^{20}$ program. All the structures were solved by direct methods and refined on $F^{2}$ by full-matrix least squares techniques with the SHELXTL ${ }^{21}$ software package. All non-H atoms were anisotropically refined. Hydrogen atoms were added according to the theoretical models. Atomic scattering factors and anomalous dispersion corrections were taken from International Tables for XRay Crystallography. ${ }^{22}$

Crystallographic data (excluding structure factors) for the structure reported in this article have been deposited with the Cambridge Crystallographic Data Centre as supplementary publication CCDC no. 647812 for the compound $\mathrm{C}_{16} \mathrm{H}_{15} \mathrm{~N}_{3} \mathrm{O}_{2}$. Copy of the data can be obtained free of charge on application to CCDC, 12 Union Road, Cambridge CB1 1EZ, UK (Fax: t44-1223/336-033; e-mail: deposit@ccdc.cam. ac.uk).

\subsection{Antibacterial activity}

The antibacterial activities of the synthesized compounds was tested against $B$. subtilis, $S$. aureus, $S$. faecalis, E. coli, P. aeruginosa and E. cloacae using MH medium. The MICs of the test compounds were determined by a colorimetric method using the dye MTT. ${ }^{23}$ A stock solution of the synthesized compound $(50 \mu \mathrm{g} / \mathrm{mL})$ in DMSO was prepared and graded quantities of the test compounds were incorporated in specified quantity of sterilized liquid $\mathrm{MH}$ medium. A specified quantity of the medium containing the test compound was poured into microtitration plates. Suspension of the microorganism was prepared to contain approximate $10^{5} \mathrm{cfu} / \mathrm{mL}$ and applied to microtitration plates with serially diluted compounds in DMSO to be tested and incubated at $37^{\circ} \mathrm{C}$ for $24 \mathrm{~h}$. After the MICs were visually determined on each of the microtitration plates, $50 \mu \mathrm{L}$ of PBS containing $2 \mathrm{mg}$ of MTT/mL was added to each well. Incubation was continued at room temperature for $4-5 \mathrm{~h}$. The content of each well was removed, and $100 \mu \mathrm{L}$ of isopropanol containing $5 \% 1 \mathrm{~mol} / \mathrm{L}$ $\mathrm{HCl}$ was added to extract the dye. After $12 \mathrm{~h}$ of incubation at room temperature, the optical density (OD) was measured with a microplate reader at $550 \mathrm{~nm}$. The observed MICs were presented in table 2.

\section{Results and discussion}

\subsection{Synthesis}

There are two isomers of $1 \mathrm{H}-$ and $2 \mathrm{H}$-benzotriazoles in solid state equilibrium (scheme 1). While $2 \mathrm{H}$ substituted perhaps surprisingly being of the same order of stability as $1 \mathrm{H}$-substituted and often the isomers $1 \mathrm{H}$ - and $2 \mathrm{H}$-substituted show the same reactions. ${ }^{12}$ Mannich bases were prepared by the Mannich reaction of substituted acetophenone and benzotriazole ${ }^{13}$ and two isomers, viz. $1 \mathrm{H}-$ and $2 \mathrm{H}-$ substituted (scheme 1), were found in the products, and were isolated by column chromatography, with the former being the majority (the yield is more than $90 \%$ ). The proportion of these two isomers depended on substituent on the acetophenone molecule and the reaction conditions. Acetophenones with electrondonating group on benzene ring gave more $2 \mathrm{H}$ substituted product than those with electronwithdrawing substituted group. The same result was observed when the reaction temperature is lower. Even if under its best conditions, the yield of the $2 \mathrm{H}$-substituted is still low, always less than 10 percent. The $1 \mathrm{H}-$ and $2 \mathrm{H}$-substituted products were isolated by column chromatography and identified from each other by solution ultraviolet spectra or $\mathrm{X}$-ray single-crystal diffraction analyses. It seems that $1 \mathrm{H}$-substituted product show an absorption band at $250 \mathrm{~nm}$ with broad absorption extended to $330 \mathrm{~nm}$, whereas 2-benzotriazole derivatives show one absorption band at $250 \mathrm{~nm}$ and other structures band at $280 \mathrm{~nm}$ (figure 1). The structure of the isomers can be obtained using X-ray single-crystal diffraction analyses (figures 2 and 3 ).

The synthesis of compounds $\mathbf{1 - 2 7}$ is illustrated in scheme 1. 1H-Benzotriazolylpropanone and $2 \mathrm{H}-$ benzotriazolylpropanone were prepared according to 


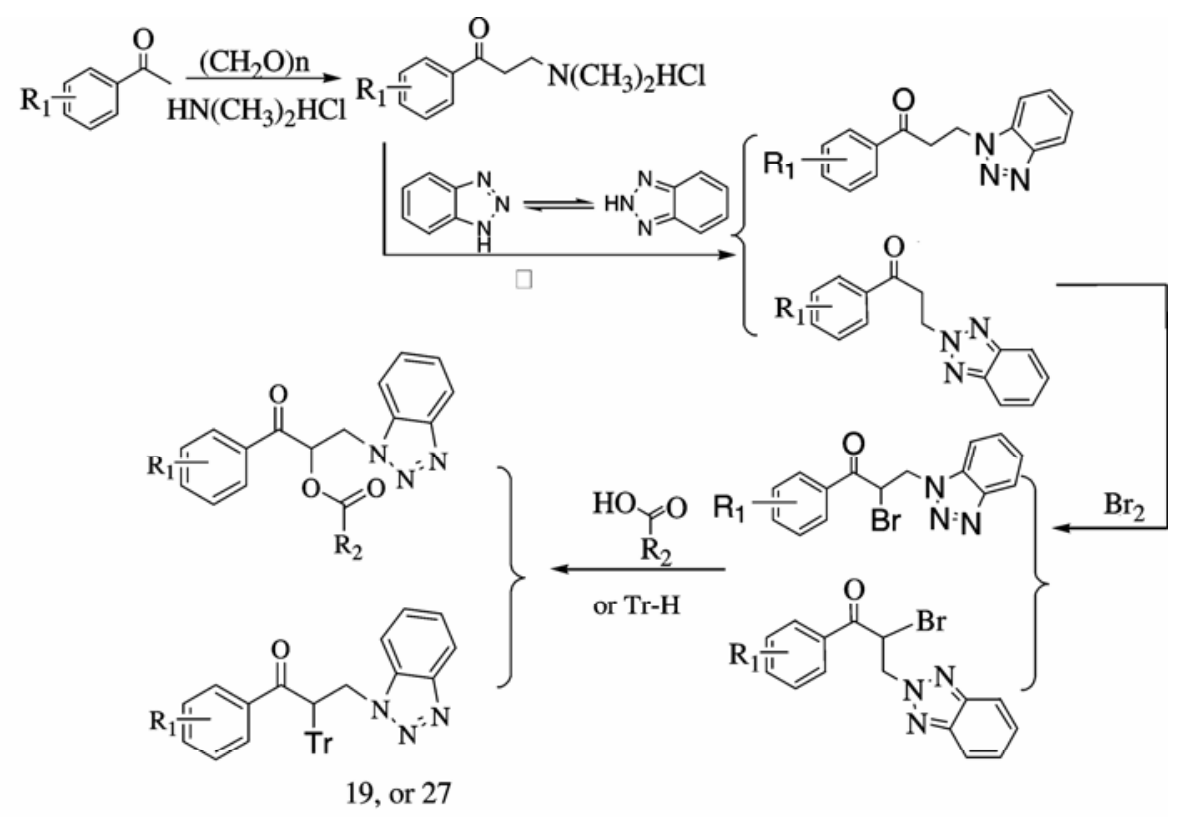

1: $\mathrm{R}_{1}=3-\mathrm{OCH}_{3}, \mathrm{R}_{2}=3$-pyridine;

2: $\mathrm{R}_{1}=3-\mathrm{OCH}_{3}, \mathrm{R}_{2}=4$-pyridine;

3: $\mathrm{R}_{1}=3-\mathrm{OCH}_{3}, \mathrm{R}_{2}=$ phenyl;

4: $\mathrm{R}_{1}=3-\mathrm{OCH}_{3}, \mathrm{R}_{2}=2$,4-dichlorophenyl;

5: $\mathrm{R}_{1}=3-\mathrm{OCH}_{3}, \mathrm{R}_{2}=2$-chlorophenyl;

6: $\mathrm{R}_{1}=4-\mathrm{CH}_{3}, \quad \mathrm{R}_{2}=$ phenyl;

7: $\mathrm{R}_{1}=4-\mathrm{CH}_{3}, \quad \mathrm{R}_{2}=2$,4-dichlorophenyl;

8: $\mathrm{R}_{1}=4-\mathrm{CH}_{3}, \quad \mathrm{R}_{2}=4$-ethylphenyl;

9: $\mathrm{R}_{1}=4-\mathrm{CH}_{3}, \quad \mathrm{R}_{2}=4$-chlorophenyl;

10: $\mathrm{R}_{1}=4-\mathrm{CH}_{3}, \quad \mathrm{R}_{2}=2$-chlorophenyl;

11: $\mathrm{R}_{1}=3-\mathrm{Cl}, \quad \mathrm{R}_{2}=2$-chlorophenyl;

12: $\mathrm{R}_{1}=3-\mathrm{Cl}, \quad \mathrm{R}_{2}=4$-pyridine;

13: $\mathrm{R}_{1}=4-\mathrm{Br}, \quad \mathrm{R}_{2}=3$-pyridine;
14: $\mathrm{R}_{1}=4-\mathrm{Br}, \quad \mathrm{R}_{2}=4$-pyridine;

15: $\mathrm{R}_{1}=4-\mathrm{Br}, \quad \mathrm{R}_{2}=4$-chlorophenyl;

16: $\mathrm{R}_{1}=4-\mathrm{Br}, \quad \mathrm{R}_{2}=2$-chlorophenyl;

17: $\mathrm{R}_{1}=4-\mathrm{Br}, \quad \mathrm{R}_{2}=p$-tolyl;

18: $\mathrm{R}_{1}=4-\mathrm{Br}, \quad \mathrm{R}_{2}=o$-tolyl;

19: $\mathrm{R}_{1}=4-\mathrm{Br}, \mathrm{Tr}$;

20: $\mathrm{R}_{1}=2-\mathrm{CH}_{3}, \quad \mathrm{R}_{2}=3$-pyridine;

21: $\mathrm{R}_{1}=2-\mathrm{CH}_{3}, \quad \mathrm{R}_{2}=4$-pyridine;

22: $\mathrm{R}_{1}=2-\mathrm{CH}_{3}, \quad \mathrm{R}_{2}=4$-chlorophenyl;

23: $\mathrm{R}_{1}=2-\mathrm{CH}_{3}, \quad \mathrm{R}_{2}=2$-chlorophenyl;

24: $\mathrm{R}_{1}=2-\mathrm{CH}_{3}, \quad \mathrm{R}_{2}=p$-tolyl;

25: $\mathrm{R}_{1}=2-\mathrm{CH}_{3}, \quad \mathrm{R}_{2}=4$-ethylphenyl;

26: $\mathrm{R}_{1}=2-\mathrm{CH}_{3}, \quad \mathrm{R}_{2}=o$-tolyl;

27: $\mathrm{R}_{1}=2-\mathrm{CH}_{3}, \mathrm{Tr}$

Scheme 1. Synthesis of compounds 1-27.

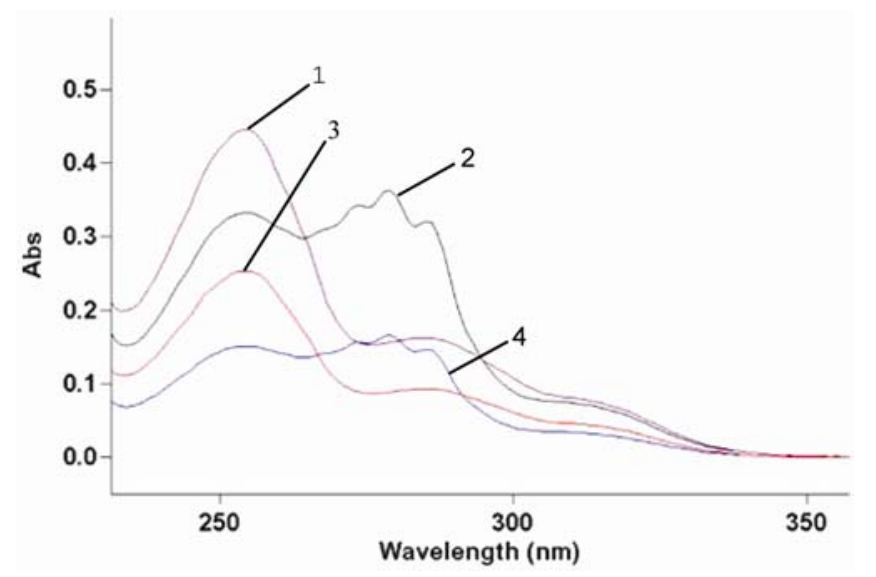

Figure 1. The UV spectrum of different concentration of 3-(benzo[1,2,3]triazol-yl)-1-(3-methoxyphenyl)-1-oxopropan derivatives.

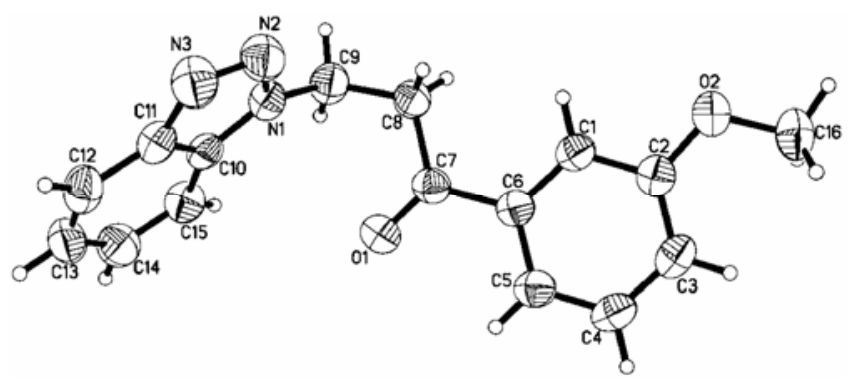

Figure 2. Molecular structure for the $1 \mathrm{H}$-substituted derivative of the target compounds.

the reported methods. ${ }^{15}$ Bromine substituted benzotriazolylpropanone was obtained in the presence of sodium acetate and acetic acid when the ratio of $1 \mathrm{H}$-benzotriazolylethanones and sodium acetate was $1: 2$, the reaction temperature is 
decreased to $30^{\circ} \mathrm{C}$ and furnished the monobromide. Using triethylamine as the binding acid reagent, compounds 1-27 were obtained at room temperature, in good yields ${ }^{16-18}$ (scheme 1). After the succeeding brominating reaction of these products, the substitution reaction of the $1 \mathrm{H}$-substituted products is very easy, but the reaction of the $2 \mathrm{H}$-substituted products is quite a hard progress.

\subsection{Description of the crystal structure}

Figures 4 and 5 show the molecular structure of compound 1 and its packing arrangements in unit cells. The summary of the crystal data, experimental details and structure refinement for compound $\mathbf{1}$ are listed in table 1 . The bond lengths and angles fall within normal ranges, ${ }^{19}$ and are comparable with those in each of the compound. Most bond lengths in the molecules are between the single and double bonds, which shows high $\pi$-electron delocalized and a large conjugated system formed. The bond lengths of $\mathrm{C}-\mathrm{N}$ are in the range of $1.357-1.453 \AA$, shorter

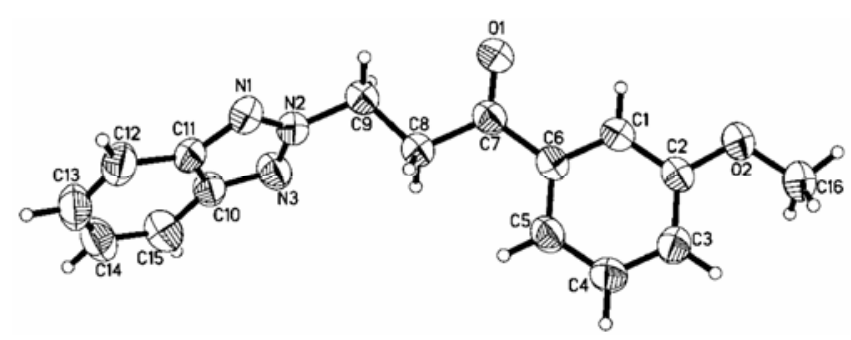

Figure 3. Molecular structure for the $2 \mathrm{H}$-substituted derivative of the target compounds. (CCDC no. is 647812).

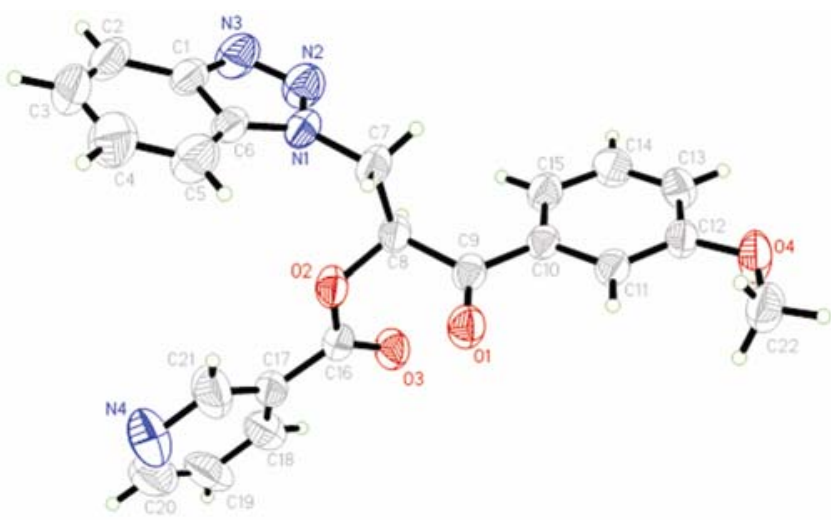

Figure 4. The X-ray crystal structure of compound $\mathbf{1}$ with the atomic numbering scheme drawn at a $50 \%$ probability level. than the single bond length of $1.48 \AA$ and longer than the typical $\mathrm{C}=\mathrm{N}$ distance of $1.28 \AA$, indicating

Table 1. Crystal data and structure refinement parameters for 1 .

\begin{tabular}{ll}
\hline Compound & \multicolumn{1}{c}{1} \\
\hline Formula & $\mathrm{C}_{22} \mathrm{H}_{8} \mathrm{~N}_{4} \mathrm{O}_{4}$ \\
Formula weight & $402 \cdot 40$ \\
Crystal size $\left(\mathrm{mm}^{3}\right)$ & $0 \cdot 06 \times 0 \cdot 21 \times 0 \cdot 43$ \\
Crystal system & Monoclinic \\
Wavelength $(\mathrm{MoK} \alpha)(\AA)$ & $0 \cdot 71073$ \\
Space group & $P 2_{1} / c$ \\
$A(\AA)$ & $9 \cdot 960(2)$ \\
$b(\AA)$ & $9 \cdot 551(2)$ \\
$c(\AA)$ & $20 \cdot 803(4)$ \\
$\alpha\left({ }^{\circ}\right)$ & 90 \\
$\beta\left(^{\circ}\right)$ & $90 \cdot 252(4)$ \\
$\gamma\left({ }^{\circ}\right)$ & 90 \\
$V\left(\AA^{3}\right)$ & $1953 \cdot 2(7)$ \\
$Z, D_{\text {calcd }}\left(\mathrm{mg} / \mathrm{m}^{3}\right)$ & $4,1 \cdot 368$ \\
$F(000)$ & 840 \\
$\left.\mu(\mathrm{mm})^{-1}\right)$ & $0 \cdot 097$ \\
$\theta$ ranges $\left({ }^{\circ}\right)$ & $2 \cdot 0-26 \cdot 1$ \\
Temperature $(\mathrm{K})$ & $293(2)$ \\
Refl. Collected/unique & $10630 / 3853$ \\
Data/parameters & $2853 / 271$ \\
Max./min. transmission & $0 \cdot 9596 / 0 \cdot 9942$ \\
Final $R$ indices $[I>2 \sigma(I)]$ & $R_{1}=0 \cdot 0505, w R_{2}=0 \cdot 1174$ \\
$R$ indices $($ all data) & $R_{1}=0 \cdot 0753, w R_{2}=0 \cdot 1309$ \\
Goodness-of-fit on $F^{2}$ & $1 \cdot 038$ \\
Max./min., $\Delta \rho$ & $0 \cdot 204 /-0 \cdot 213$ \\
CCDC No. & 672175 \\
\hline &
\end{tabular}

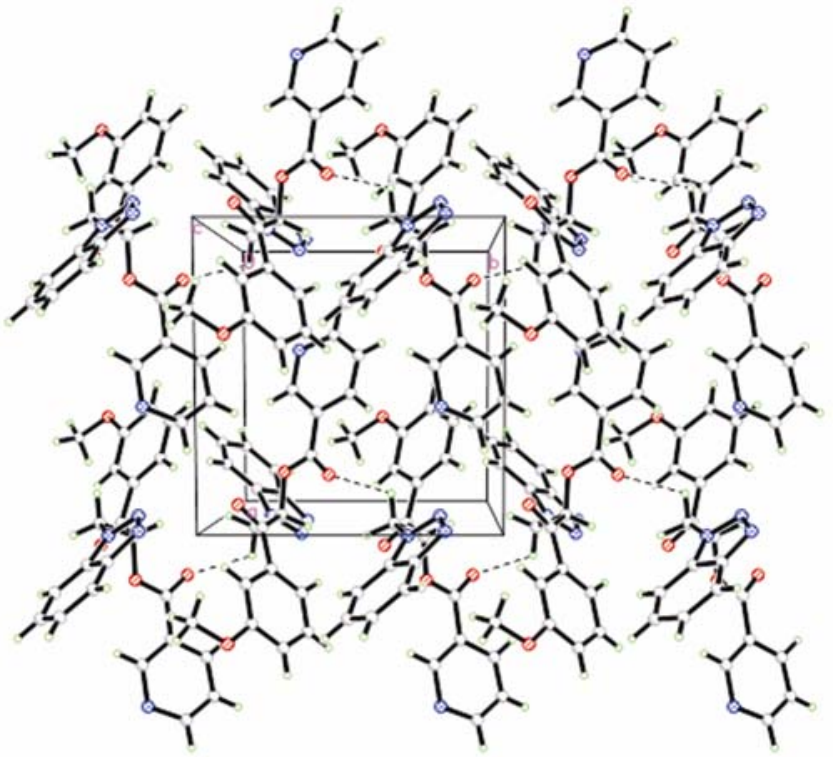

Figure 5. Packing digagram of 1 showing the intermolecular hydrogen bonds (dishes lines), viewed down the $c$ axis. 
Table 2. Antibacterial activity of the selected compounds.

\begin{tabular}{lcccccc}
\hline & \multicolumn{5}{c}{ Minimum inhibitory concentrations $(\mu \mathrm{g} / \mathrm{mL})$} \\
\cline { 2 - 7 } Compounds & B. subtilis & S. aureus & S. faecalis & P. aeruginosa & E. coli & E. cloacae \\
\hline $\mathbf{1}$ & $12 \cdot 5$ & 25 & $6 \cdot 25$ & $6 \cdot 25$ & 25 & $12 \cdot 5$ \\
$\mathbf{2}$ & $12 \cdot 5$ & $>50$ & $6 \cdot 25$ & $6 \cdot 25$ & $6 \cdot 25$ & $>50$ \\
$\mathbf{3}$ & $>50$ & $>50$ & 25 & $>50$ & $>50$ & $>50$ \\
$\mathbf{4}$ & $1 \cdot 56$ & $3 \cdot 12$ & $3 \cdot 12$ & $12 \cdot 5$ & $3 \cdot 12$ & $12 \cdot 5$ \\
$\mathbf{5}$ & $6 \cdot 25$ & $>50$ & $>50$ & $>50$ & $>50$ & $6 \cdot 25$ \\
$\mathbf{6}$ & $12 \cdot 5$ & $12 \cdot 5$ & $6 \cdot 25$ & $>50$ & $6 \cdot 25$ & $12 \cdot 5$ \\
$\mathbf{7}$ & $1 \cdot 56$ & $6 \cdot 25$ & $>50$ & $12 \cdot 5$ & $12 \cdot 5$ & $>50$ \\
$\mathbf{8}$ & $6 \cdot 25$ & $12 \cdot 5$ & $6 \cdot 25$ & $>50$ & $>50$ & $12 \cdot 5$ \\
$\mathbf{9}$ & $6 \cdot 25$ & $>50$ & $>50$ & $12 \cdot 5$ & $6 \cdot 25$ & $>50$ \\
$\mathbf{1 0}$ & $12 \cdot 5$ & $3 \cdot 12$ & $12 \cdot 5$ & $12 \cdot 5$ & $>50$ & $12 \cdot 5$ \\
$\mathbf{1 1}$ & $>50$ & 25 & $>50$ & $>50$ & 25 & 25 \\
$\mathbf{1 2}$ & 25 & $>50$ & $>50$ & $>50$ & $>50$ & $12 \cdot 5$ \\
$\mathbf{1 3}$ & $12 \cdot 5$ & 25 & $6 \cdot 25$ & $6 \cdot 25$ & 25 & $12 \cdot 5$ \\
$\mathbf{1 4}$ & $12 \cdot 5$ & $>50$ & $6 \cdot 25$ & $6 \cdot 25$ & $6 \cdot 25$ & $>50$ \\
$\mathbf{1 5}$ & $12 \cdot 5$ & 25 & $6 \cdot 25$ & $>50$ & $3 \cdot 12$ & $12 \cdot 5$ \\
$\mathbf{1 6}$ & $1 \cdot 56$ & $3 \cdot 12$ & $1 \cdot 56$ & $>50$ & 25 & $6 \cdot 25$ \\
$\mathbf{1 7}$ & $3 \cdot 12$ & $6 \cdot 25$ & 25 & $3 \cdot 12$ & $6 \cdot 25$ & 25 \\
$\mathbf{1 8}$ & $1 \cdot 56$ & $1 \cdot 56$ & $12 \cdot 5$ & $12 \cdot 5$ & 25 & $12 \cdot 5$ \\
$\mathbf{1 9}$ & $1 \cdot 56$ & $1 \cdot 56$ & $1 \cdot 56$ & $3 \cdot 12$ & $6 \cdot 25$ & $6 \cdot 25$ \\
$\mathbf{2 0}$ & 25 & $>50$ & $>50$ & $>50$ & 25 & $12 \cdot 5$ \\
$\mathbf{2 1}$ & $>50$ & $>50$ & 25 & $>50$ & $6 \cdot 25$ & $>50$ \\
$\mathbf{2 2}$ & $>50$ & $12 \cdot 5$ & 25 & $12 \cdot 5$ & $3 \cdot 12$ & $12 \cdot 5$ \\
$\mathbf{2 3}$ & $6 \cdot 25$ & $12 \cdot 5$ & $>50$ & $12 \cdot 5$ & 25 & $6 \cdot 25$ \\
$\mathbf{2 4}$ & $3 \cdot 12$ & 25 & $3 \cdot 12$ & 25 & $3 \cdot 12$ & 25 \\
$\mathbf{2 5}$ & $3 \cdot 12$ & 25 & $6 \cdot 25$ & 25 & 25 & 25 \\
$\mathbf{2 6}$ & $1 \cdot 56$ & $6 \cdot 25$ & $6 \cdot 25$ & $12 \cdot 5$ & $6 \cdot 25$ & $6 \cdot 25$ \\
$\mathbf{2 7}$ & $>50$ & $>50$ & 25 & 25 & $6 \cdot 25$ & $12 \cdot 5$ \\
Penicillin & $1 \cdot 56$ & $1 \cdot 56$ & $1 \cdot 56$ & $6 \cdot 25$ & $6 \cdot 25$ & $3 \cdot 12$ \\
Kanamycin & $0 \cdot 39$ & $1 \cdot 56$ & $3 \cdot 12$ & $3 \cdot 12$ & $3 \cdot 12$ & $6 \cdot 25$ \\
\hline
\end{tabular}

partial double character. This can be interpreted in terms of conjugation in the heterocyclic. The C7C8-C9 angle is $113.99(18)^{\circ}$ for 1 , respectively, determined by $s p^{3}$ hybridization state of $\mathrm{C} 8$ atom. The benzotriazole moieties are essentially planar, with dihedral angles of $0.99(13)^{\circ}$ between the triazole ring and $\mathrm{C} 1-\mathrm{C} 6$ benzene ring, and make dihedral angles of $62.35(9)^{\circ}$ with the $\mathrm{C} 10-\mathrm{C} 15$ benzene rings in 1, respectively. In addition, the dihedral angle between the N4/C17-C21 pyridine ring and the plane benzotriazole system is $82.77(12)^{\circ}$. The results show that the benzotriazole and pyridine molecule are not plane. In the solid state, one carboxyl oxygen atom of nicotinic acid forms an intermolecular hydrogen bond with the carbon atom of another compound $\mathbf{1}$, the donor and acceptor distance $\mathrm{C} 7 \ldots \mathrm{O} 3$ is $3.005 \AA$ (symmetry code: $-x$, $1 / 2+y, 1 / 2-z]$, the whole molecules are linked into infinite chains along the $\mathrm{c}$ axis by intermolecular hydrogen bonds.

\subsection{Antibacterial assay in vitro}

All the compounds prepared were evaluated for their antibacterial activities against three Gram positive bacterial strains (B. subtilis, S. aureus and $S$. faecalis) and three Gram negative bacterial strains (E. coli, P. aeruginosa and E. cloacae) activities by MTT method, and the results are shown in table 2 .

As showed in table 2, compounds 4, 7, 16, 18, 19 and 26 exhibited significant activity with MIC values of $1.56 \mu \mathrm{g} / \mathrm{mL}$ against $B$. subtilis, which were comparable to the positive control penicillin. Compounds 17, 24 and 25 showed moderate activity with MIC values of $3 \cdot 12 \mu \mathrm{g} / \mathrm{mL}$ against $B$. subtilis. A comparison of the substitution on benzotriazoylpropanone demonstrated halogen substitution on the benzotriazoylpropanone showed higher antibacterial activity against $B$. subtilis. This result suggested that the introduction of halogen substituent increased the 
hydrophobicity of the synthesized compounds and lead to the increase of the antibacterial activity.

Compounds 18 and 19 exhibited significant activity with MIC values of $1.56 \mu \mathrm{g} / \mathrm{mL}$ against $S$. aureus, which were comparable to the positive control penicillin. Compounds 4, 10 and 16 showed moderate activity with MIC values of $3.12 \mu \mathrm{g} / \mathrm{mL}$ against $S$. aureus.

Besides, compounds 16 and 19 displayed potent activity with MIC values of $1.56 \mu \mathrm{g} / \mathrm{mL}$ against $S$. faecalis, which were comparable to the positive control penicillin, Compounds $\mathbf{4}$ and $\mathbf{2 4}$ showed moderate activity with MIC values of $3 \cdot 12 \mu \mathrm{g} / \mathrm{mL}$ against $S$. faecalis. Other compounds showed activity against $S$. faecalis, with the MIC values of $6 \cdot 25-$ $50 \mu \mathrm{g} / \mathrm{mL}$. From table 2 we can also find that compounds 4, 15, 22 and 24 showed significant activity with MIC values of $3.12 \mu \mathrm{g} / \mathrm{mL}$ against $E$. coli, which were comparable to the positive control kanamycin, and compounds 2, 6, 9, 14, 17, 19, 21, 26 and 27 exhibited moderate activity against $E$. coli with MIC values of $6 \cdot 25 \mu \mathrm{g} / \mathrm{mL}$. Compounds 17 and 19 exhibited significant activity with MIC values of $3 \cdot 12 \mu \mathrm{g} / \mathrm{mL}$ against $P$. aeruginosa, which were comparable to the positive control kanamycin. Besides, Compounds 5, 16, 19, 23 and 26 exhibited activity with MIC values of $6.25 \mu \mathrm{g} / \mathrm{mL}$ against E. cloacae, which were comparable to the positive control kanamycin. The mechanism of antibacterial activity of the synthesized benzotriazole derivatives is under study, and will be reported in due course.

\section{Conclusion}

In this paper, a series of benzotriazole derivations were synthesized and their chemical structures were confirmed by means of ${ }^{1} \mathrm{H}$ NMR, IR and elemental analyses, and one of them was determined by single crystal X-ray diffraction analysis. The compounds were assayed for antibacterial (B. subtilis, S. aureus, $S$. faecalis, $P$. aeruginosa, E. coli and E. cloacae) activities by MTT (3-(4,5-dimethylthiazol-2-yl)-2,5diphenyl tetrazolium bromide) method. Among the compounds tested, compound 3-Benzotriazol-1-yl-1(4-bromo-phenyl)-2-[1,2,4]triazol-1-yl-propan-1-one (19) showed the most favourable antibacterial activity against $B$. subtilis, $S$. aureus, $S$. faecalis, $P$. aeruginosa, E. coli and E. cloacae with MIC of $1.562 \mu \mathrm{g} / \mathrm{mL}, 1.562 \mu \mathrm{g} / \mathrm{mL}, 1.562 \mu \mathrm{g} / \mathrm{mL}, 3.125 \mu \mathrm{g} /$ $\mathrm{mL}, 6.25 \mu \mathrm{g} / \mathrm{mL}$ and $6.25 \mu \mathrm{g} / \mathrm{mL}$ respectively. Further SAR and mechanistic studies on this new class of compounds are currently under active investigation and will be reported in due course.

\section{Acknowledgements}

This work was supported by the Natural Science Foundation of Shandong Province (No. Y2007B50), the Foundation of Key Laboratory of Organofluorine Chemistry, Shanghai Institute of Organic Chemistry, Chinese Academy of Sciences (O4B0021400), and Outstanding Adult-young Scientific Research Encouraging Foundation of Shandong Province (2008BS09017).

\section{References}

1. Katarzyna K, Zelika N and Justyna Z 2004 Bioorg. Med. Chem. 122617

2. Li H-Q, Xu C, Li H-S, Xiao Z-P, Shi L and Zhu H-L 2007 Chem. Med. Chem. 21361

3. Biagi G, Giorgi I, Livi O, Scartoni V, Barili P L and Calderone V 2001 Il Farmaco 56827

4. Scapin G, Patel S B, Becker J W, Wang Q, Desponts C, Waddleton D, Skorey K, Cromlish W, Bayly C, Therien M, Gauthier JY, Li C-S, Lau C K, Ramachandran C, Kennedy B P and Asante-Appiah E 2003 Biochemistry 4211451

5. Antonio C, Michele P, Gianpiero B, Bernardetta B, Marta M and Roberta L 2004 Il Farmaco 59637

6. Xu L-Z, Jian F-F, Gao H-R and Chong C-Y 2003 Chin. J. Chem. 211615

7. Bushuev M B, Virovets A V, Garcia Y, Gieck C, Sheludyakova L A, Ikorskii V N, Tremel W, Gütlich P and Lavrenova L G 2002 Polyhedron 21797

8. Touami S M, Poon C C and Wender P A $1997 \mathrm{~J} . \mathrm{Am}$. Chem. Soc. 1197611

9. C Y Wu, King K Y, Kuo C J, Fang J M, Wu Y-T, Ho M Y, Liao C-L, Shie J J, Liang P-H and Wong C H 2006 Chem. Biol. 13261

10. Handratta V D, Vasaitis T S, Njar V C O, Gediya L K, Kataria R, Chopra P, Newman D, Farquhar R, Guo Z, Qiu Y and Brodie A M H 2005 J. Med. Chem. 482972

11. Tomás F, Catalán J, Pĕrez $\mathrm{P}$ and Elguero J 1994 J. Org. Chem. 592799

12. Wang H, Burda C, Persy G and Wirz J $2000 \mathrm{~J}$. Am. Chem. Soc. 1225849

13. Abonia R, Insuastya B, Quirogaa J, Noguerasb M and Meierc H 2004 Mini-reviews in organic chemistry, 1 387

14. Zeng W-L, Bi S, Zhong $\mathrm{H}$ and Wan J 2006 Acta Cryst. E62 O5801

15. Zhang S-S, Wan J, Li C-L, Li X-M and Qu B 2007 J. Heterocyclic Chem. $\mathbf{4 4} 75$

16. Wan J, Peng Z-Z, Li X-M and Zhang S-S 2006 Acta Cryst. E62 O4410 
17. Bi S, Zeng W L and Wan J 2007 Acta Cryst. E63 $\mathrm{O} 1078$

18. Zeng W L, Bi S and Wan J 2007 Acta Cryst. E63 O1076

19. Allen F H, Kennard O, Watson D G, Brammer L, Orpen A G and Taylor R 1987 J. Chem. Soc. Perkin Trans. 2 S1

20. SADABS 1996 Program for empirical absorption correction of area detector data (Germany: University of Gottingen)
21. Sheldrick G M 1996 SHELXTL, v5 Reference Manual, Siemens Analytical X-ray Sysytems, Inc., Madison, Wisconsin, USA

22. Wilson A J C 1992 International table for X-ray crystallography (Dordrecht: Kluwer Academic Publishers), volume C, tables 6.1.1.4, pp. 500-502 and 4.2 .6 .8 , pp. $219-222$ respectively.

23. Meletiadis J, Meis J F, Mouton J W, Donnelly J P and Verweij P E 2000 J. Clin. Microbiol. 38 2949 